\title{
FOREGROUND OBJECT EXTACTION BASED ON INDEPENDENT COMPONENT ANALYSIS
}

\author{
Rahul Paul $^{1}$ and Sushanta Mukhopadhyay ${ }^{2}$ \\ ${ }^{1}$ Department of Computer Engineering, ISM Dhanbad,India \\ ${ }^{2}$ Department of Computer Engineering, ISM Dhanbad,India
}

\begin{abstract}
Moving objects are often characterized by coherent motion that is distinct from that of the background. This makes motion a very useful feature for segmenting video sequence. Extracting moving objects from videos is important for many applications like surveillance, traffic analysis etc. In this paper a novel and efficient moving object segmentation algorithm is proposed that is based on independent component analysis (ICA). Moving objects and static background are considered to be independent, so independent component analysis is applied on frames of a video sequence to identify the preliminary independent components containing moving objects. This source image data obtained after ICA are further processed using anisotropic diffusion. Anisotropic diffusion is used here to reduce the noise present in the preliminary source image without removing the significant parts of the image content. Finally, a post-processing step based on morphology is applied on the obtained objects to remove small unnecessary objects and to smooth the object boundary to produce the final segmented images indicating the moving objects. The method is tested on various datasets and experimental results establish the satisfactory performance of the proposed algorithm.
\end{abstract}

\section{KEYWORDS}

Motion segmentation, Independent component analysis, anisotropic diffusion, mathematical morphology

\section{INTRODUCTION}

Detection of moving objects in a video sequence is an essential step for video evaluation. The increasing popularity of video analysis is due to the high demand for video in security related applications, traffic analysis, research and education etc. Segmentation is the key step for analyzing the moving objects in a video. Segmentation is a processing where frames obtained from of a video are treated as input and the output is much simpler version where homogeneous parts are partitioned into simple relevant segments. There are some methods proposed for segmentation of moving objects $[7,8]$. These methods can be roughly classified into two broad categories: foreground extraction based and background construction based video object segmentation. In foreground extraction approach, temporal, spatial or both spatio-temporal is first used to obtain an initial video object. Then using the motion information in the successive frames the moving object can be identified and separated. In contrast to background construction based approach, foreground extraction approach can segment the moving object more efficiently with a more specific object boundary.

In background construction approach, the background image is first created and then an initial video object is obtained based on the difference on the background and current frame. This method is suitable for fast moving objects and its computational cost is also low. 
Advances in Vision Computing: An International Journal (AVC) Vol.2, No.4, December 2015

In this paper we are interested in challenging sequences containing complex motions, and sudden change in the background. Here we propound independent component analysis (ICA) along with anisotropic diffusion and morphological operators for segmenting the moving objects. Independent component analysis is a signal processing technique in which the goal is to find a linear representation of non-Gaussian data so that the components are statistically independent. Such a representation is very useful in many applications including feature extraction and signal separation. Du Ming Tsai [1] has applied ICA for background subtraction for indoor surveillance. They have combined ICA along with particle swarm optimization (PSO)in their approach. Another proposition based on contour and ICA based segmentation is proposed by Hicham Sekkati [2]. Xiao-Ping Zhang [3] presented an approach for video object extraction based on spatio-temporal analysis. Zhong Zhang [9] proposed a method- Contextual Constrained Independent Component Analysis (CCICA) that tends to detect slowly moving objects. In this method contextual constraints are explicitly added to the optimization object function which indicates the similarity relationship between neighbouring pixels.

In our algorithm, ICA is first used to extract the independent components from linearly mixed signals. Results in ICA domain generally based on two assumptions: a) the source signals are independent of each other and $b$ ) the values in each source signals have non-gaussian distributions. The obtained components are processed using anisotropic diffusion. It is an iterative procedure which allows noise removal and enhancement of sharp features simultaneously. Finally morphological operators are used for smoothing object edges and removing small unnecessary objects.

The organization of this paper is as follows. The various steps proposed method is presented in section 2. Section 2, 1 first elucidates the ICA model. Section 2.1.1, 2.1.2 and 2.1.3 analyzed Kurtosis, negentropy and pre-processing methods for ICA process respectively. Section 2.2 describes anisotropic diffusion method by Perona and Malik. Mathematical morphological operations are explained in section 2.3. Proposed segmentation approach is elucidated in section 2.4. Section 3 demonstrates the results obtained using the suggested method. Section 4 expounds the conclusion of the paper.

\section{LITERATURE SURVEY}

The detection of interesting and moving foreground object from a video provides a classification of the pixels into either foreground or background. When the scene is stationary or gradually evolving, the foreground object detection can be done using many traditional background subtraction algorithms. One of the important work in the frame differencing method has done by Jain and Nagel[1979] . However background subtraction became popular following the work of Wren et al. [1997]. Elgammal and Davis [2000] use nonparametric kernel density estimation to model the background . Li and Leung[2002] combine the texture and color features to perform the background subtraction. One of the famous temporal modeling method known as Mixture of Gaussians (MoG) proposed by Stauffer and Grimson [10]. In this method a pixel in the current frame is checked against the background model by comparing it with every Gaussian in the model until a match is found. For image segmentation and clustering approach Comaniciu and Meer[2002] proposed the mean shift approach to find the clusters in the joint spatial and color space. Horn and Schunk in give the example of a rotating sphere with no surface markings which, under constant illumination, causes no changes in the image intensity over time. Cheng et al. proposed a DWT based process which depends on inter frame differencing for multiple objects tracking and identification. Huang (2004) [11] proposed a new wavelet based moving object segmentation method based on three consecutive frames $I_{n-1}, I_{n}$ and $I_{n+1}$.Baradarani et al. (2006) developed a multi wavelet based approach for moving object segmentation and detection. Du Ming Tsai[1] apply ICA for background subtraction for indoor surveillance. They apply ICA 
along with particle swarm optimization (PSO) in their approach. Another proposition based on contour and ICA based segmentation is proposed by Hicham Sekkati [2]. Xiao-Ping Zhang [3] presented an approach for video object extraction based on spatiotemporal ICA analysis and multiscale segmentation.

\section{PROPOSED METHOD}

The proposed method employs the following tools.

\subsection{Independent Component Analysis}

ICA is a powerful technique in which the goal is to retrieve independent signals from a mixture of signals, and the independent signals must have some unknown non Gaussian distribution. Aapo Hyvärinen[4] deerived FASTICA, an efficient and fast algorithm for independent component analysis .

In the basic ICA model, each instance of the variable $\mathrm{x}_{\mathrm{j}}$ is expressed as a linear mixtures $\mathrm{x}_{1}, \mathrm{x}_{2}, \ldots, \mathrm{x}_{\mathrm{n}}$ of $\mathrm{n}$ independent components.

$$
x_{j}=a_{j 1} s_{1}+a_{j 2} s_{2}+\ldots .+a_{j n} s_{n}, \text { for all } j
$$

In the matrix notation, $\mathrm{x}=\mathrm{As}$, where $\mathrm{A}=\left[\begin{array}{ccc}a 11 & \cdots & a 1 n \\ \vdots & \ddots & \vdots \\ a j 1 & \ldots & a j n\end{array}\right]$

$\mathrm{s}$, represents the mutually independent source signals. Now the goal of ICA is to estimate some matrix $\mathrm{W}$, so that $\mathrm{s}(\mathrm{t})=\mathrm{Wx}$. This means the solution to ICA would be to find $\mathrm{W}\left(\mathrm{W}=\mathrm{A}^{-1}\right)$. Main idea behind estimating independent componenets is through non-gaussianity. In practice there are several different measures of non-gaussianity and here we are explaining Kurtosis and Negentropy.

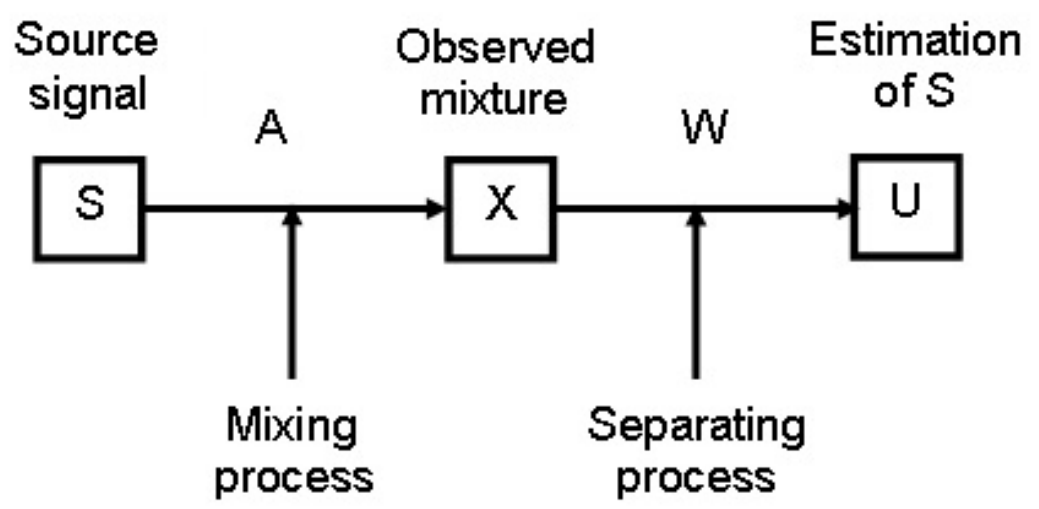

Fig. 1 ICA method

\subsubsection{Kurtosis}

The main approach to find non-gaussianity is kurtosis or the fourth order moment, defined as

$$
\operatorname{Kurt}(\mathrm{x})=\mathrm{E}\left(\mathrm{x}^{4}\right)-3\left\{\mathrm{E}\left(\mathrm{x}^{2}\right)\right\}^{2}
$$

As the variable $\mathrm{x}$ is assumed to be standard normal variable we can say

$$
\operatorname{Kurt}(y)=E\left(y^{4}\right)-3
$$


Advances in Vision Computing: An International Journal (AVC) Vol.2, No.4, December 2015

\subsubsection{Negentropy}

Negentropy is another very important approach for finding of non-gaussianity. Negentropy $\mathbf{J}$ is defined as,

$$
\mathrm{J}(\mathrm{x})=\mathrm{H}\left(\mathrm{x}_{\text {gauss }}\right)-\mathrm{H}(\mathrm{x})
$$

where, $\mathrm{x}_{\text {gauss }}$ is a gaussian random variable of the same covariance matrix as $\mathrm{x}$.

\subsubsection{Preprocessing for FASTICA \\ 3.1.3.1. Centering}

This is the preliminary and important preprocessing which is used to center the variable ' $x$ ', i.e. subtract its mean vector $\mathrm{m}$ to make $\mathrm{x}$ a zero-mean variable.

\subsubsection{Whitening}

This is another additional preprocessing technique in ICA which is used to first whiten the detected variable by converting its covariance matrix to a unit or identity matrix.

\subsubsection{FASTICA algorithm}

In our approach we apply FASTICA algorithm based on negentropy. The FASTICA algorithm process a lot of sampling point of the observation signal $\mathrm{x}$, so its rate of convergence is high. The steps of the FASTICA algorithm are:

1. Centering and whitening the mixed input signal $x$

2. Initialization and updation of orthogonal matrix $w$ in terms of previous components using $\mathrm{w}^{+}=\mathrm{E}\left\{\mathrm{xg}\left(\mathrm{w}^{\mathrm{T}} \mathrm{x}\right)\right\}-\mathrm{E}\left\{\mathrm{g}^{\prime}\left(\mathrm{w}^{\mathrm{T}} \mathrm{x}\right)\right\} \mathrm{w}$

3. $\mathrm{w}=\mathrm{w}^{+} /\left\|\mathrm{w}^{+}\right\|$. (Normalization of $\mathrm{w}$ )

4. if not converge go back to step 2 converged if norm $\left(w_{\text {new }}-w_{\text {old }}\right)>\xi$. Value of $\xi$ is typically around 0.0001 .

\subsection{Anisotropic Diffusion}

Diffusion describes the movements of particles from higher density regions to lower density regions. There are two types of diffusion- Isotropic diffusion (not dependent of direction) and anisotropic diffusion (depends on the direction applied). Anisotropic diffusion proposed by Perona \& Malik, is a technique for reducing image noise without removing significant parts of the image content, typically edges, lines or other details that are important for the interpretation of image. Nonlinear partial differential equations (PDE) are applied in the nonlinear diffusion method to create a scale space representation that comprises of moderately simplified images .Isotropic diffusion is an instance of the heat equation as a PDE, given as:

$$
\frac{\partial I}{\partial t}=\nabla^{2} I=\frac{\partial^{2} I}{\partial x^{2}}+\frac{\partial^{2} I}{\partial y^{2}}
$$

where, $\mathrm{I}=$ image taken and $\mathrm{t}=$ time of evolution and $\mathrm{x}, \mathrm{y}$ are the spatial co-ordinates. 
Perona \& Malik[5] presented the anisotropic diffusion. They introduce the flux function as a means to constrain the diffusion to contiguous homogeneous regions, but not cross region boundaries. The heat equation is this modified to:

$$
\frac{\partial I}{\partial t}=c(x, y, t) \Delta I+\nabla c \cdot \nabla I
$$

where, $\mathrm{c}$ is the suggested flux function which guides the rate of diffusion at any point in the image.

Perona \& Malik proposed two flux functions as:

$$
\begin{aligned}
& c(\|\nabla I\|)=e^{-(\|\nabla I\| / K)^{2}} \\
& c(\|\nabla I\|)=\frac{1}{1+\left(\frac{\|\nabla I\|}{K}\right)^{2}}
\end{aligned}
$$

The flux functions proffer a quid pro quo between edge-preservation and blurring homogeneous regions. $\mathrm{k}$ is the free parameter which manage both the functions and it also decides the strength of an edge to reckon as a rational boundary of a particular region. Larger value of $k$ will give us a solution same as isotropic diffusion. The process is allowed to continue till an acceptable convergence.

\subsection{Mathematical Morphology}

Morphological image processing employs a group of operations with prime importance to the shape or morphology of characteristics in an image. In morphological process, we probe an image with a kernel of small shape and size called structuring element, which defines the region of interest or neighborhood around a pixel. The fundamentals morphological operators are erosion, dilation, opening and closing as illustrated below.

\subsubsection{Erosion}

The erosion of the image $\mathrm{f}$ by a flat structuring element $\mathrm{B}$ at any spatial location $(\mathrm{x}, \mathrm{y})$ is defined as the minimum value of the image in the region coincident with $\mathrm{B}$ and defined by the shape and size of $\mathrm{B}$, when the origin of $\mathrm{B}$ is at $(\mathrm{x}, \mathrm{y})$ and is given by :

$$
[f \ominus B](x, y)-\min _{(s, t) \in B}\{f(x+s, y+t)\}
$$

\subsubsection{Dilation}

Dilation is the other fundamental operations in morphological image processing. The dilation of image $\mathrm{f}$ by a flat structuring element $\mathrm{B}$ at any location $(\mathrm{x}, \mathrm{y})$ is defined as the maximum value of the image in the window outlined by B when the origin of B is at $(\mathrm{x}, \mathrm{y})$. That is

$$
[f \oplus B](x, y)=\max _{(a, t) \varepsilon B}\{f(x-s, y-t)\}
$$


Advances in Vision Computing: An International Journal (AVC) Vol.2, No.4, December 2015

\subsubsection{Opening and Closing}

In image processing closing and opening are the basic processes of morphological noise removal. Closing detaches small holes whereas opening separates small objects.

For binary image, Opening is the dilation of the erosion of a image $f$ by a structuring element $B$

$$
f \circ B=[(f \ominus B) \oplus B]
$$

and closing of a set A by a structuring element B is the erosion of the dilation of that set.

$$
f \cdot B=[(f \oplus B) \ominus B]
$$

\subsection{Proposed Approach}

The ICA expansion, $\mathrm{x}=$ ws

1. To get $\mathrm{x}$, change each frame into a vector by lexicographic scanning.

2. Generate a series of images which share some common information but changing other fixed parts

3. center the images to make its mean zero and then whiten the images.

4. Apply FASTICA algorithm

5. Convert the ICs to images

6. Sensitive to the position change

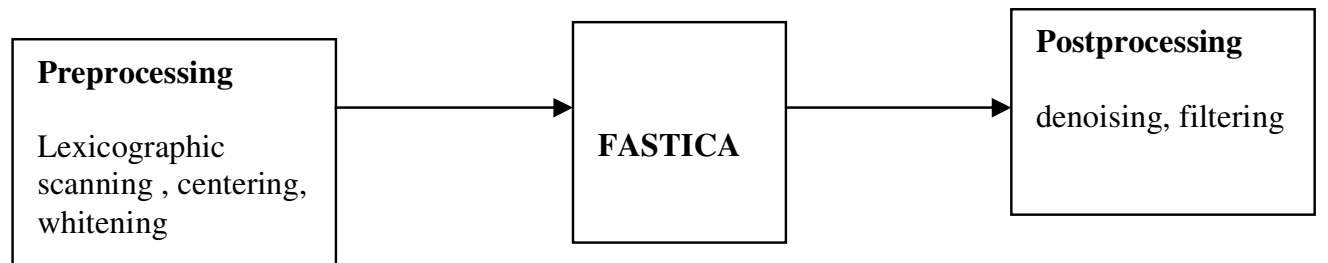

Fig. 2 Fundamental procedures implemented and exploited in the FASTICA for efficient decomposition

In some of the video frames the motion is lower whereas in some of the frames the motion is higher with reference to time. Now plot the retrieved components and choose only those components which have lower motion corresponding to static background. Anisotropic diffusion resembles the approach that creates scale space, where an image generates a parameterized family of successively more and more blurred images based on a diffusion process. Anisotropic diffusion is now applied on the selected segmented images to reduce noise and keeping significant parts. Finally morphological operations are used over the diffused component images.

Initially a threshold is chosen and the small objects whose area is below the threshold value are discarded. Now morphological closing and erosion is applied on the image obtained after applying threshold. 


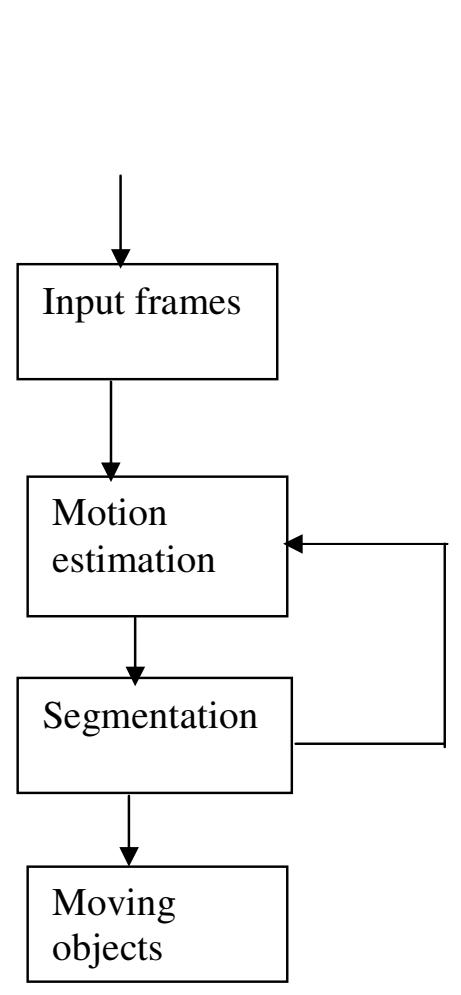

(a)

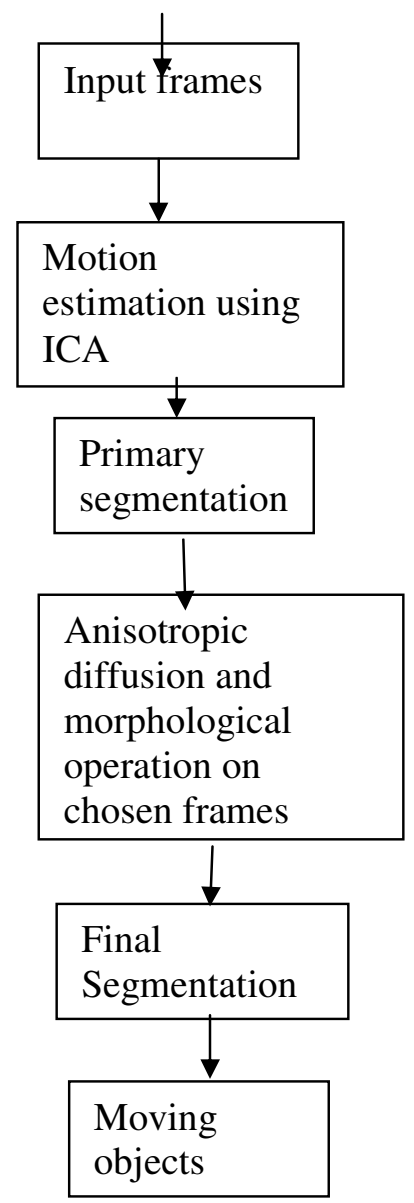

(b) 


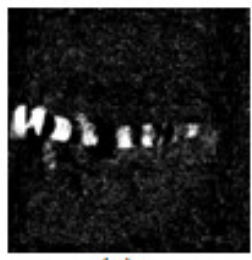

(a)

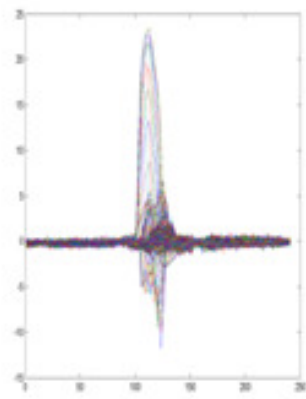

(e)

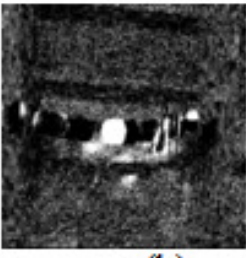

(b)

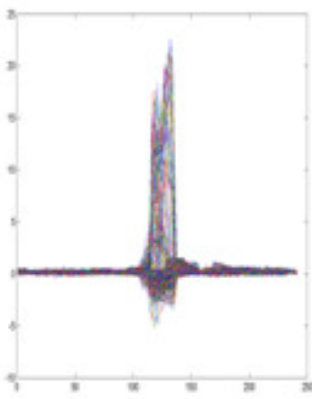

(f)

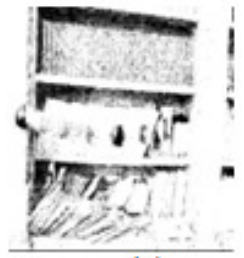

(c)

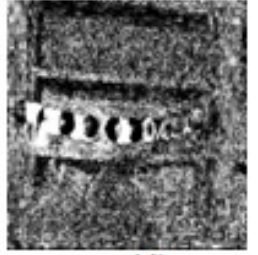

(d)

Fig. 3 (a) Simple motion based segmentation and (b) Segmentation using proposed method 


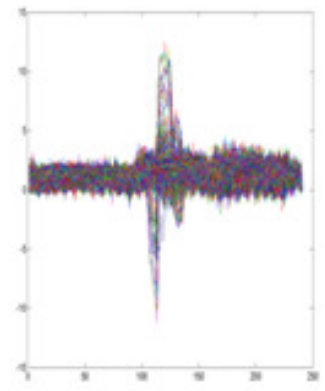

(h)

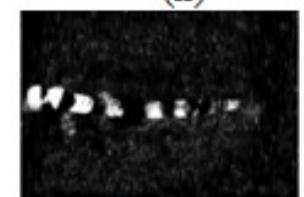

(i)

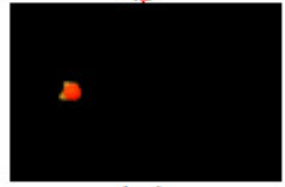

(m)

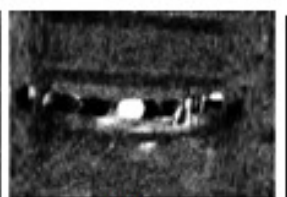

(j)

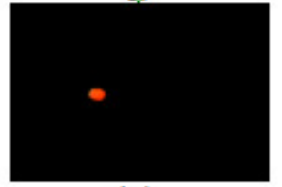

(n)

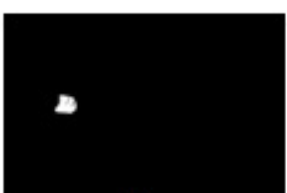

(k)

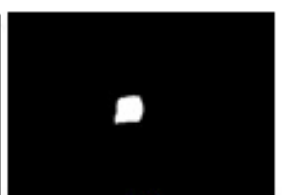

(1)

Fig. 4 (a-d) independent components; (e-h) plots of independent components ; (i,j) diffused image of independent components a \& b respectively; $(\mathrm{k}, \mathrm{l})$ objects after applying morphological operation; $(\mathrm{m}, \mathrm{n})$ final segmentation result

\section{EXPERIMENTAL RESULTS}

In this section, we present the experimental results from two sets of both outdoor sequence and indoor sequence with varying lighting condition to evaluate the performance of the proposed ICA based moving object extraction method. Instead, a comparison with fusion schemes using GMM and optical flow are performed. The video taken for our approach are in avi and mpg formats with a frame size of $240 \times 320$ pixels. The performance of the proposed method runs at a fast speed for $240 \times 320$ images on a personal computer with an AMD 32 bit $2.2 \mathrm{GHz}$ processor. For indoor sequence first dataset is of Intelligent Room: a person is moving room one side to another. Second dataset of indoor sequence is a moving ball. In outdoor sequence first dataset is about a moving car and second dataset is walking: person walking in a corridor.

\subsection{First Dataset}

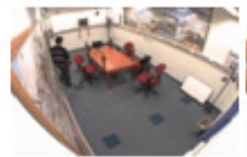

(a) frame 91

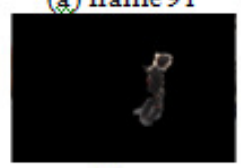

(f)

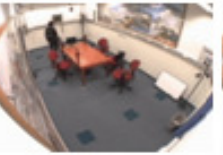

(b) frame 122

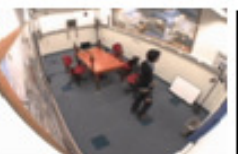

(c) frame 290

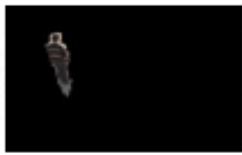

(d)

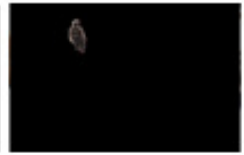

(e) 


\subsection{Second Dataset}

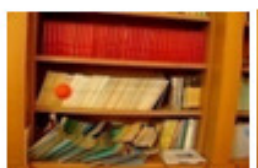

(a) frame 10

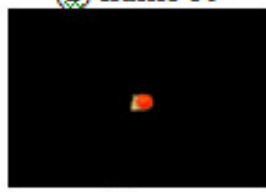

(f)

\subsection{Third Dataset}

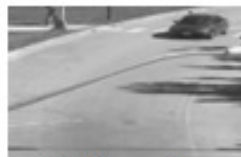

(a) frame 3

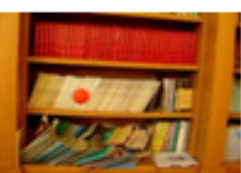

(b) frame 14

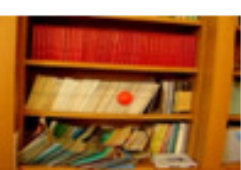

(c) frame 57

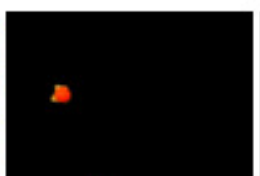

(d)

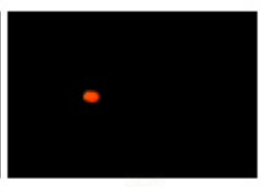

(e)

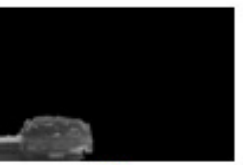

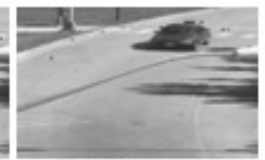

(b) frame 15

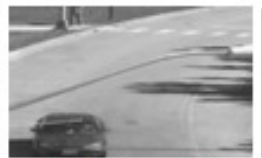

(c) frame 88

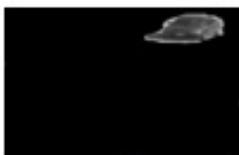

(d)

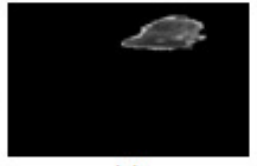

(e)

(f)

\subsection{Fourth Dataset}

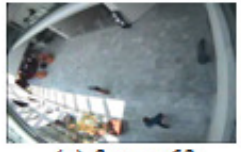

(a) frame 63

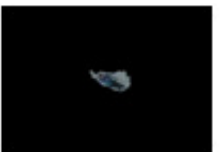

(f)

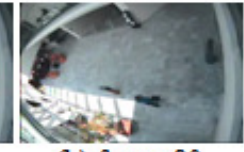

(b) frame 80

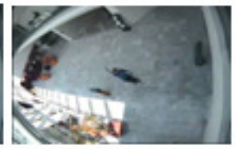

(c) frame 120

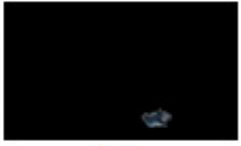

(d)

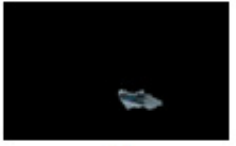

(e)

Fig. 5 Segmentation results obtained using proposed method

\section{Conclusions}

Each moving object contains some unique character which differentiates one from another and that can be used for pattern analysis using object recognition and segmentation. Depending on the study of independent components on an image sequence a new moving object segmentation approach using improved ICA is proposed in this paper. The importance of this paper lies in the fact that the new method uses ICA to process image sequences directly. The experimental results show that the new algorithm has the advantages of simple computation with faster processing 
Advances in Vision Computing: An International Journal (AVC) Vol.2, No.4, December 2015

speed. We can find in some results that the proposed segmentation approach over-segments the moving object. The algorithm can be used for numerous applications with accurate results and has great flexibility.

\section{REFERENCES}

[1] D.M Tsai and S.C. Lai, "Independent component analysis-based background subtraction for indoor surveillance,” IEEE Trans. Image Processing, 18( 1), (2009).

[2] H. Sekkati, R. Laganiere, A. Mitiche and R. Youmaran "Robust background subtraction using geodesic active contours in ICA subspace for video surveillance applications," IEEE Conference on Computer and Robot Vision, pp. 190-197, (2012) .

[3] X. Zhang and Z. Chen, "An automated video object extraction system based on spatiotemporal independent component analysis and multiscale segmentation," EURASIP Journal on Applied Signal Processing, (2006), pp. 1-22

[4] A.Hyvaerinen and E. Oja, "Independent component analysis: algorithms and applications," Neural Networks, 13(4-5), pp. 411-430, 2000.

[5] P. Perona and J.Malik , "Sacle-space and edge detection using anisotropic diffusion," IEEE trans. PAMI, 12(7), pp. 629-639

[6] J. Weickert , "Anistropic Diffusion in image processing” Teubner, Sttutgart,(1998)

[7] A.Yilmaz, O.Javed and M. Shah, "Object tracking: a survey," ACM Computing Surveys, vol. 38, no. 4, December 2006.

[8] M. Piccardi, "Background subtraction techniques: a review," IEEE International Conference on Systems, Man and Cybernetics, vol. 4, pp. 3099-3104 .

[9] Z. Zhang, B Xiao, C Wang, W. Zhou and S. Liu , "Contextual constrained independent component analysis based foreground detection for indoor surveillance," Pattern Recognition(ACPR), pp. 701705, November 2011.

[10] C. Stauffer and W. Grimson, "Learning patterns of activity using real-time tracking," IEEE Trans. Pattern Analysis and Motion Intelligence, vol. 22, pp. 747-757, 2000 .

[11] J.C.Huang,T.S Su, L.J Wang and W.S. Hsieh, "Double change detection method for wavelet based moving object segmentation," Electronics Letters , vol. 40, no. 13 , 2004

\section{Authors}

Rahul Paul is a student at the Indian School of Mines, Dhanbad, India. He received B.Tech degree in Computer Science \& Engineering from WBUT, WestBengal, India. He has done his M.Tech in Computer Science \& Engineering from Indian School of Mines, Dhanbad, India. His research area and interest include image processing, video processing and object tracking.

Sushanta Mukhopadhyay is currently an Associate Professor, in the Department of Computer Science \& Engineering, ISM Dhanbad. He did his m.tech in Radiophysics and Electronics from the University of Kolkata and completed his Ph.D in Image Processing from ISI,Kolkata. During 2001-03 and 2004-07 he worked at the Burnham Institute,California,Usa and NTU,Singapore respectively. His research area and interest include image processing, fMRI, image compression, image encryption and image watermarking.
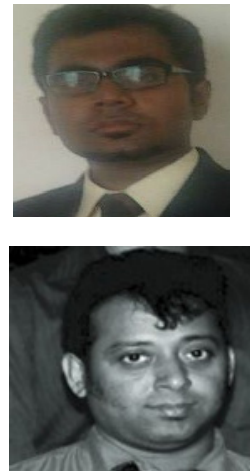